\title{
Typhoons Affecting Taiwan: Current Understanding and Future Challenges
}

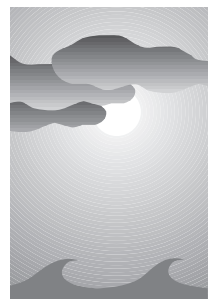

Chun-Chieh $\mathrm{Wu}^{*}$ and Ying-Hwa $\mathrm{KuO}^{+}$

\begin{abstract}
Of all the natural disasters occurring in Taiwan, tropical cyclones are the most serious. Over a 20-yr period, Taiwan was hit by an average of 3.7 typhoons per year. These storms can produce heavy rainfall and strong winds, leading to severe damage to agriculture and industry, and serious loss of human life. An outstanding example is Typhoon Herb, which made landfall in Taiwan on 31 July 1996. Typhoon Herb took 70 lives and caused an estimated $\$ 5$ billion of damage to agriculture and property.

Accurate prediction of the track, intensity, precipitation, and strong winds for typhoons affecting Taiwan is not an easy task. The lack of meteorological data over the vast Pacific Ocean and the strong interaction between typhoon circulation and Taiwan's mesoscale Central Mountain range are two major factors that make the forecasting of typhoons in the vicinity of Taiwan highly challenging. Improved understanding of the dynamics of typhoon circulation and their interaction with the Taiwan terrain is needed for more accurate prediction. With this objective in mind, the National Science Council in Taiwan sponsored the Workshop on Typhoon Research in the Taiwan Area at Boulder, Colorado, on 17-18 May 1997. In this paper, the authors review the observational and numerical studies of typhoons affecting Taiwan, present some preliminary results from the study of Typhoon Herb, summarize the recommendations obtained from the workshop, and provide suggestions for future research.
\end{abstract}

\section{Introduction}

Tropical cyclones are the most serious meteorological phenomena that threaten Taiwan. On average, three or four typhoons occur annually. The strong winds and heavy precipitation associated with a typhoon significantly affect the weather in Taiwan, even if the storm does not make landfall. Due to the high altitude and complicated mesoscale topography associated with Taiwan's Central Mountain range (CMR), which has an average elevation of about $3000 \mathrm{~m}$ and a dimension of $300 \mathrm{~km} \times 100 \mathrm{~km}$, significant variations in track and intensity occur as a typhoon approaches the island. [Note that similar effects also

\footnotetext{
*Department of Atmospheric Sciences, National Taiwan University, Taipei, Taiwan.

${ }^{+}$National Center for Atmospheric Research, Boulder, Colorado. Corresponding author address: Dr. Chun-Chieh Wu, Department of Atmospheric Sciences, National Taiwan University, 61, Lane 144, Section 4, Keelung Rd., Taipei 10772, Taiwan.

E-mail: cwu@typhoon.as.ntu.edu.tw

In final form 8 September 1998.

(C) 1999 American Meteorological Society
}

occur for tropical storms approaching other island terrains, such as the islands of Cuba, Hispaniola, and Puerto Rico in the Caribbean, as well as Luzon in the northern Philippines (Bender et al. 1987).]

The interaction of the typhoon circulation with the CMR also produces significant mesoscale variations in pressure, wind, and precipitation distribution over Taiwan. For example, mesoscale secondary low centers can form on the lee side of the CMR, which may develop into a new tropical cyclone, replacing the original storm. The track deflection, the development of secondary lows, the changes in intensity, and the mesoscale structure of pressure, wind, and precipitation associated with an approaching typhoon make the forecasting of a tropical cyclone approaching Taiwan an extremely difficult task. The understanding of the dynamics of typhoon circulation and their interaction with the Taiwan terrain, and the forecasting of track, intensity, and mesoscale wind and precipitation distribution are some of the most important scientific and forecasting problems for meteorologists in Taiwan.

The recent occurrence of Typhoon Herb between 31 July and 1 August 1996 was a special example of a 
typhoon making landfall on Taiwan. This storm produced a maximum 24-h rainfall of $1798 \mathrm{~mm}$ over the CMR. Although the track forecast by the Central Weather Bureau (CWB) in Taiwan was essentially correct, the unusually strong winds and torrential precipitation associated with Herb caused tremendous damage. In particular, the extremely heavy rainfall in the central mountain areas triggered disastrous flooding, debris flows, and landslides, which led to the loss of 51 human lives, with an additional 22 people missing and presumed dead. The total damage to agriculture and property is estimated at more than $\$ 5$ billion. Thus, Typhoon Herb replaced Typhoon Thelma (1977) as the most disruptive weather event on record in Taiwan.

In view of the impact of typhoons on the lives and the economy of Taiwan, the Fifth National Science and Technology Conference (Taipei, September 1996) decided to launch a national project for disaster mitigation, under which an integrated program of both basic and forecasting research into typhoons was established. In order to provide a forum for discussion on typhoon research and forecasting, and to stimulate international collaboration, the National Science Council of Taiwan sponsored the Workshop on Typhoon Research in the Taiwan Area on 17-18 May 1997 at the National Center for Atmospheric Research in Boulder, Colorado. The objectives of the workshop, which was attended by 60 scientists, were 1) to review the current state of science on basic and forecasting research into tropical cyclones, 2) to identify critical research and forecasting issues for typhoons affecting Taiwan, and 3) to discuss opportunities for international collaboration on typhoon research.

The purpose of this paper is to discuss the current understanding of research and forecasting issues related to typhoons affecting Taiwan and to summarize the discussion that took place at the workshop. Section 2 provides a brief review of previous studies on typhoons affecting Taiwan. For a thorough review of the effects of Taiwan topography on typhoons, see Lin (1993). Preliminary analysis and model simulation of Typhoon Herb are given in section 3 to highlight the research and forecasting issues. Section 4 provides a summary of the Workshop on Typhoon Research in the Taiwan Area. Suggestions for future research are given in section 5 .

\section{Review of previous studies on typhoons affecting Taiwan}

a. Observational studies

When a typhoon approaches Taiwan, its circula- tion and track are significantly affected by the CMR. Brand and Blelloch (1974) found that westbound typhoons tend to move cyclonically around the northern side of the CMR and experience an average intensity decrease of over $40 \%$, beginning about $12 \mathrm{~h}$ before the storm centers reach the island. Using streamline analysis, Chu et al. (1977) showed a more detailed flow structure, such as flow separation and vortex shedding, associated with typhoon circulation around the island. Wang (1980) documented and analyzed the path, intensity, propagation speed, and evolution of 53 typhoons that approached Taiwan over the period 1946-75. He found that some typhoons passed over Taiwan by taking a cyclonic track similar to those described in Brand and Blelloch (1974). Based on these observations, Wang (1980) proposed a conceptual model, indicating that the typhoon center will be deflected toward the north as it approaches Taiwan and toward the south after its passage over the CMR due to the deflection of the mean steering flow upstream of the CMR.

For a typhoon impinging on the Taiwan CMR, Wang $(1980,1989)$ also showed that the center of the storm could either cross Taiwan continuously or have a discontinuous track due to the formation of a secondary low that replaces the terrain-blocked low-level center. Wang (1980) proposed that, for typhoons with a continuous track, the circulation is only modified slightly by the mountain range, while for typhoons with a discontinuous track, it is modified significantly. This seems to be consistent with nonlinear theories of stratified flow over a mountain (e.g., Smith 1979) since typhoons with a continuous track have stronger winds associated with them and tend to be more linear because the local Froude number associated with the mountain is larger. This is also related to the contrast in intensity between the two classes of typhoons (Chang 1982).

The CMR affects not only the track of a typhoon, but also its structure. A recent observational study (Shieh et al. 1996) has categorized four possible flow regimes as a typhoon approaches Taiwan: 1) the parallel flow regime, where the flow field associated with a typhoon basically moves along the CMR with a small incident flow angle, which is defined as the flow direction relative to the main axis of the CMR; 2) the blocked flow regime, where the incident angle is quite large and the Froude number (Fr) is low; 3) the unblocked (upsloping) flow regime, which is the same as 2) but with a relatively high Fr; 4) the combined flow regime with low-level flow passing around, but upper-level flow crossing over, the mountain. Wang 
(1989) suggested that the factors determining the above flow regimes include the typhoon's heading direction, the Froude number, the typhoon incident angle, and the location of the typhoon center.

It should be noted that comprehensive archives of raw observational data for typhoons affecting Taiwan are located in the "Typhoon Data Bank" of the Central Weather Bureau in Taiwan. Although some statistical results from the observations have been carried out (e.g., Lee et al. 1997), more detailed analyses of these data have yet to be conducted to further improve our understanding of the effect of Taiwan's terrain on typhoons.

An important issue associated with the observational studies of typhoons affecting Taiwan is the lack of data. Most of the previous observational studies on this subject are based on traditional surface and upperair observations, which are highly inadequate in describing the important three-dimensional structure changes associated with a typhoon as it approaches the island. Due to the lack of surface observations over the mountains and the surrounding oceans, even the surface analysis of an approaching storm can be subject to numerous uncertainties, not to mention the difficulty in accurately describing the three-dimensional storm structure. Mesoscale observations from groundbased and airborne Doppler radars and other remote sensing instruments are needed to advance the observational studies.

\section{b. Numerical studies}

1) IDEALIZED FLOW OVER TOPOGRAPHY

The interaction between a typhoon and topography is highly complicated. To gain insight into such a challenging problem, it is important to review relevant literature on the dynamics of airflow passing a mountain.

During the past three decades, much effort has been devoted to the study of the dynamics of uniform flow past a mountain. Smith (1989a) proposed a linear theory to predict the occurrence of stagnation points as a function of hill shape and ambient shear. For a long ridge perpendicular to a weakly sheared flow, stagnation begins aloft. Stagnation aloft is believe to be responsible for gravity wave breaking and severe downslope winds (Clark and Peltier 1977). For a ridge aligned with the flow, waves weaken dispersively aloft and stagnation first occurs on the surface. This allows density surfaces to intersect the ground and the lowlevel flow to split around the hill. As a ridge is aligned more in parallel with wind direction, the critical mountain height for the occurrence of stagnation both upwind and aloft increases, but the critical mountain height for stagnation aloft increases more rapidly. This is because, in a stratified flow, the vertically propagating waves weaken aloft due to dispersion.

The salient features in the response of a densitystratified fluid passing a three-dimensional obstacle at low Fr are the existence of a flow reversal zone on the windward side and intense vertically oriented vortices on the lee side of the obstacle. The generation of lee vortices was explained as a consequence of the separation of the viscous boundary layer from the obstacle (Batchelor 1967). However, Smolarkiewicz and Rotunno (1989, SR hereafter) argued that lee vortices can be produced even in inviscid numerical simulations. They proposed that the vertically oriented vorticity of lee vortices is generated through the tilting of horizontally oriented vorticity that is produced baroclinically as isentropes deform in response to the flow over the obstacle. Smolarkiewicz and Rotunno believed that the lee vortices are closely associated with the dynamics of nonlinear gravity waves and the conservation of potential vorticity (PV). However, Smith (1989b) argued that the onset of stagnation points might result in regions with collapsed density surfaces and/or convective overturning and turbulence. These processes can lead to PV generation, which explains the formation of lee vortices.

Wang et al. (1997) conducted systematic numerical experiments to study the formation mechanisms for the stagnation points in a three-dimensional flow over idealized bell-shaped mountains with different aspect ratios. The flow regimes, especially the critical Froude number for the occurrence of stagnation points, have been investigated. Their findings are 1) the upwind stagnation is a necessary rather than a sufficient condition for the generation of lee vortices; 2) both the upwind blocking effects and lee deceleration in the central portion of the obstacle are suppressed as the obstacle elongates in the direction along the mean flow; 3 ) the major role of the nonlinear terms is to decelerate the zonal flow in the central portion of the lee surface; 4) the vertically propagating gravity waves and at higher levels are suppressed by the nonlinear terms. The diagnosis of the sources of the vertically oriented vorticity and PV (see Wu and Kurihara 1996) on the lee side of the obstacle is being carried out and may help clarify the above discrepancies in the lee vortices' formation mechanisms.

\section{2) TYPHOONS OVER THE CMR}

Several numerical studies have been conducted to investigate the effects of the CMR on track modifica- 
tion. Using a primitive-equation model, Chang (1982) showed that, due to blocking effects, the mountaininduced flow deflections are mainly confined to the lower levels, and the typhoon's passage induces a mean cyclonic circulation around the mountain, forcing the storm to take a cyclonic track around the northern end of the CMR. This is consistent with the conceptual model proposed by Wang (1980).

Chang (1982) also found that the secondary vortex centers form in the lee trough and may develop when they are in phase with the upper-level vortex centers. He proposed that the horizontal advection of positive vorticity, in conjunction with the lee side vortex stretching, results in a mean positive vorticity around the mountain. Later, Chan (1984) showed a definite link between the local change of relative vorticity and typhoon movement, which is consistent with the findings of Chang (1982).

Using the Geophysical Fluid Dynamics Laboratory (GFDL) hurricane model with triply nested, movable meshes, Bender et al. (1985) found that tropical cyclones fill much more rapidly in numerical simulations with a mountain included. The mountain range affects the decay rate through reduction in the supply of latent and kinetic energy into the storm circulation both during, as well as after, passage of the storm over the mountain. The mountain range also enhances the heavy precipitation to the right of the storm track immediately after landfall, which is possibly related to an increase in coastal convergence and orographic lifting.

Bender et al. (1987) also examined the effects of different island terrains on tropical cyclones under different zonal mean flows. In a control experiment with a typhoon embedded in a uniform $5 \mathrm{~m} \mathrm{~s}^{-1}$ easterly flow over Taiwan, a northward (cyclonic) track deflection was found. They suggested that the terraininduced steering flow modification was the main cause of the typhoon track deflection. In a subsequent experiment with a $10 \mathrm{~m} \mathrm{~s}^{-1}$ easterly flow, deflection was found to be much weaker. In contrast, the northward deflection was larger and began farther upstream in the case of a weaker zonal flow. This is in agreement with the results found in the aforementioned observational and numerical studies. In the simulation with a $10 \mathrm{~m} \mathrm{~s}^{-1}$ easterly flow, a secondary surface low or lows formed behind the CMR. It appears that the upper-level vortex becomes detached from the original surface low and eventually couples with the secondary one. The latent energy supply and the vertical coherence of the storm system are the important factors that determine the storm's change in intensity near and over topography. The storm is weakened by the advection of dry air from the mountain region into the storm's inner circulation and by the vertical tilting of the storm center.

Yeh and Elsberry $(1993 a, b)$ studied the upstream track deflections of westward-moving tropical cyclones approaching the mountainous terrain of Taiwan. They showed that the deflections tend to be larger for weaker and slower-moving storms, which is consistent with the findings of Bender et al. (1987). Using a primitive equation model with $45-\mathrm{km}$ horizontal resolution, two distinct regions of upstream track deflection found in observational studies were explored. As the outer circulation of the storm begins to interact with the mountain barrier, the zonal decelerations and southward track deflections are explained by an enhanced blocking and deflection of the environmental flow advecting the cyclone. The increased effect for storms approaching the southern part of the island compared to those approaching the northern part is caused by the stronger winds on the right side of the storm impinging more directly on the barrier.

Yeh and Elsberry $(1993 a, b)$ also showed that the amount of upstream track deflection and the continuity of the storm track across Taiwan depend on the storm's position relative to the CMR. In general, vortices approaching the northern part of the CMR tend to track continuously around the northern end of the island due to the deflection of the deep-layer mean flow. Discontinuous tracks predominate for vortices approaching the central and southern portions of the Taiwan topography. The numerical simulations also indicate that vortex reorganization downstream may occur as two types: 1) as a downward extension from the upper-level remnants of the typhoon; 2) as an upward growth of a lower-level secondary vortex. The first type is consistent with the modeling results of Chang (1982), while for the second type, a new lowlevel center that is separate from the original vortex or the terrain-induced pressure trough becomes the center around which the tropical cyclone reorganizes.

Smith and Smith (1995) applied the shallow-water model to the problem of a typhoon drifting past Taiwan and demonstrated that many of the observed phenomena, such as upstream blocking, downstream sheltering, corner winds, foehn, and secondary vortex formation can be simulated. Smith and Smith (1995) also found that vortex interaction with ideal topography results in a pair of trailing banners of vorticity that get wrapped up into the vortex as it drifts away. The PV production over the lee side when the cyclone ap- 
proaches the mountain is explained by the transition from an irrotational flow regime to a regime of flow over a mountain with wakes. In Smith and Smith's PV generation mechanism, new positive vorticity on the lee side is generated to the left (as one faces downstream) of the strongest wind normal to the mountain range, while in Chang's (1983) vorticity stretching mechanism, it is generated to the right of the strongest wind normal to the mountain range.

A real case simulation of Typhoon Gladys (1994) using the GFDL hurricane prediction system (Kurihara et al. 1995) was presented by Wu and Cho (1997). The control numerical experiment showed a fairly accurate track simulation for Gladys, especially during the first $54 \mathrm{~h}$. The deceleration of Gladys's translation speed as it approached the northeastern part of Taiwan and the acceleration after it passed Taiwan were shown. In general, a cyclonic track induced by the Taiwan topography was found. Although the GFDL hurricane model forecast underestimated Gladys's intensity, the model captured the evolution of Gladys's intensity, especially the weakening of Gladys during landfall. The mesoscale phenomena, including the pattern of heavy precipitation and the formation of secondary lows, were also well simulated by the model, though their locations were somewhat different from the observations.

Detailed analyses indicate that the surface low pressure center to the east of the CMR was induced by the downslope adiabatic warming (foehn) associated with the circulation of Gladys. The two quasistationary secondary vortices to the west of the CMR were mainly induced by the environmental easterly flow over the CMR, with the circulation of Gladys tending to strengthen these vortices.

Recently, Lin et al. (1997, manuscript submitted to Mon. Wea. Rev.) used a primitive-equation numerical model with prescribed latent heating to investigate the orographic influence on a propagating tropical cyclone over an idealized topography. They showed that when the cyclone approaches the mountain, orographic blocking causes the vorticity field to become asymmetric and produces new potential vorticity through turbulence and diffusion. This result suggests a new vortex generation mechanism.

\section{c. Some remaining issues}

In the above sections, various observational and modeling studies on typhoons affecting Taiwan and the interaction of typhoons with the Taiwan topography have been reviewed. Even though some progress has been made in the past two decades, many of the details of such interaction are still not well understood. Several fundamental issues have yet to be fully answered.

- The kinematics and dynamics of track deflection, as well as typhoon circulation change, under different flow regimes.

- The wake formation and vorticity generation mechanisms and their evolution.

- The physical mechanism responsible for the formation and development of the secondary lows that replace the original typhoon circulation.

- The physics of diabatic processes and the planetary boundary layer, and their impact on typhoon track, structure, and intensity when the storm passes over topography.

- The topographic effect on the mesoscale weather features, such as the distribution of wind and precipitation, associated with approaching typhoons.

In summary, the major limitations of previous research on this subject are 1) the lack of mesoscale observations that can provide a detailed description of the storms as they move across the island of Taiwan; and 2) the lack of high-resolution mesoscale model simulations that offer insights into the mesoscale processes responsible for the structural changes associated with the interaction of a typhoon with the mesoscale topography. The mesoscale observations are also very important in providing verification for model simulations so that improved models can be developed for typhoon research and prediction.

\section{Typhoon Herb (1996)}

\section{a. Synopsis of Herb}

To provide an example of typhoons affecting Taiwan we present a preliminary analysis and numerical simulation for Typhoon Herb in this section. Typhoon Herb was the eighth typhoon in the western North Pacific in 1996, and the third storm for which a typhoon warning was issued by the CWB in 1996. Herb formed at $19.9^{\circ} \mathrm{N}, 148.8^{\circ} \mathrm{E}$, about $800 \mathrm{~km}$ northeast of Guam, at 1200 UTC 24 July. As indicated from the best track analysis (Fig. 1), while intensifying, Herb moved westward in the first two days after its formation and followed the southern edge of the Pacific high. Herb then turned west-southwestward after passing $139^{\circ} \mathrm{E}$ at 0600 UTC 26 July and reached 


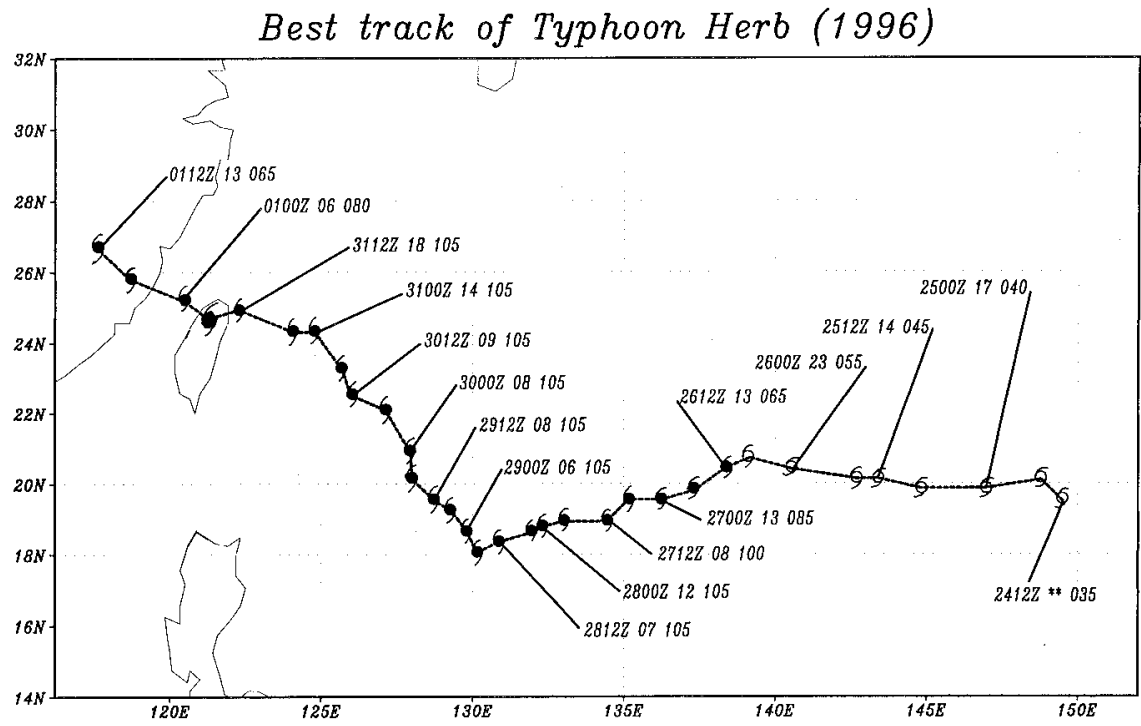

FIG. 1. Best tracks for Typhoon Herb (1996). Solid (open) storm symbol indicates best track of typhoon (tropical storm) every $6 \mathrm{~h}$. Time, translation speed (in knots), and intensity (in knots) at each time is also indicated. just south of Iriomote Shima, Japan. At 1321 UTC 31 July, about 40 min after Herb made landfall at I-Lan, RCWF recorded a few pixels with retrieved maximum wind speed of $82 \mathrm{~m} \mathrm{~s}^{-1}$ near the eyewall region at $1 \mathrm{~km}$ above sea level (Jou et al. 1997; W.-C. Lee 1998, personal communication). On Herb's way across northern Taiwan, RCWF's radar dome and antenna were destroyed.

Because of the interaction with the Taiwan terrain and the weakening of the steering flow, the center of Herb traversed northern Taiwan slowly and eventually moved off to sea near Hsin-Chu at 2100 UTC 31 July. Herb's intensity weakened sig- about $130^{\circ} \mathrm{E}$ at $1800 \mathrm{UTC} 28$ July. A maximum surface wind of $51 \mathrm{~m} \mathrm{~s}^{-1}$ and a minimum central pressure of $925 \mathrm{hPa}$ were analyzed by the CWB at 0600 UTC 27 July with an estimated gale-force-wind radius of $300 \mathrm{~km}$.

After 29 July, due to the retrogression of the Pacific high, Herb turned northwestward and headed toward the northeastern part of Taiwan with some cycloidal track oscillation. According to the best track analysis, the central pressure of Herb was about $930 \mathrm{hPa}$ during 30 July 1996 as it moved toward northern Taiwan along a westnorthwestward track. The estimated intensity of the storm decreased to $935 \mathrm{hPa}$ at $1200 \mathrm{UTC} 31$ July. Herb turned west-northwestward and made landfall at I-Lan at 1244 UTC July 31. During this period, a central pressure of $944.7 \mathrm{hPa}$ (Fig. 2) was recorded at the I-Lan station, with a gale-force-wind radius estimated at about $350 \mathrm{~km}$.

The elliptical eyewall and spiral rainbands on the western half of Herb and over northern Taiwan are well illustrated in the reflectivity factor (Fig. 3) at 0602 UTC 30 July, when Herb was about $250 \mathrm{~km}$ from the WSR-88D located over northeastern Taiwan [WuFun Shan radar station (RCWF)] and nificantly (Fig. 4) while passing across Taiwan. The measured surface wind near the storm's center dropped from 53 to $44 \mathrm{~m} \mathrm{~s}^{-1}$ in $6 \mathrm{~h}$, and the radius of gale-force wind (as estimated from satellite imagery at the CWB) also decreased from 350 to $300 \mathrm{~km}$. The Hsin-Chu station, which the center of the storm passed directly over, recorded a minimum central pressure of $968.6 \mathrm{hPa}$ (Fig. 2). The storm weakened by $24 \mathrm{hPa}$

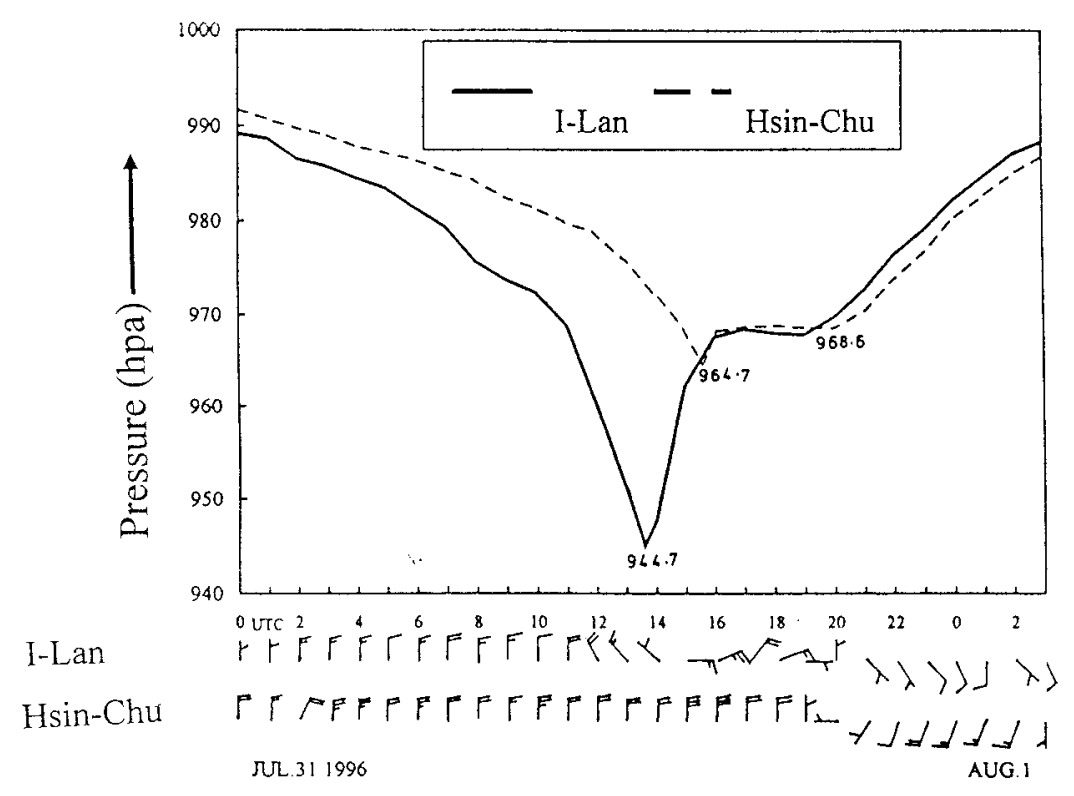

FIG. 2. Observed average surface wind (wind barb in $\mathrm{m} \mathrm{s}^{-1}$ ) and minimum central pressure at I-Lan and Hsin-Chu stations as function of time during passage of Typhoon Herb (1996) over northeastern Taiwan (after Shieh et al. 1997). 


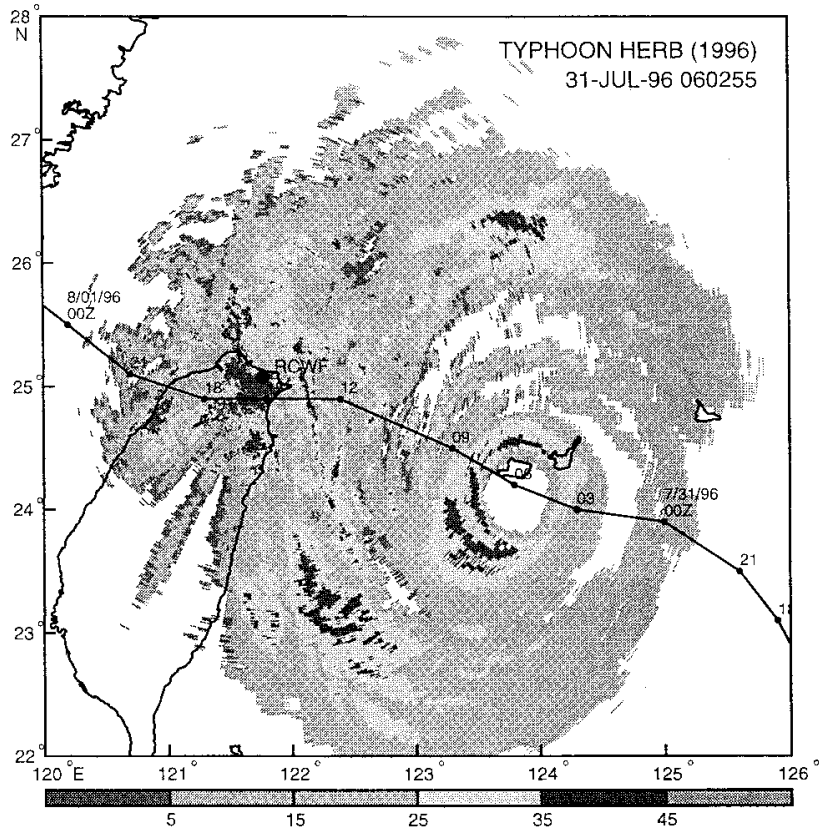

FIG. 3. Reflectivity factor (in $\mathrm{dBZ}$ ) of Typhoon Herb at $0.5^{\circ}$ PPI scan at 060255 UTC 31 July 1995 from RCWF (indicated by thick black dot in northeast corner of Taiwan). Black line shows track and position (black dots) of Typhoon Herb at 3-h intervals from 1800 UTC 30 July to 0000 UTC 1 August 1996 (W.-C. Lee 1997, personal communication).

during the 7-h period its center was over land. Finally, Herb entered the Taiwan Strait and continued tracking west-northwestward, entering southern China on 1 August.

\section{b. Rainfall and wind analysis}

A detailed analysis of Herb (S-.T. Wang 1997, personal communication) indicates several interesting mesoscale features associated with this case. The most dramatic aspect of Typhoon Herb is the enormous amount of rain it produced over the CMR (Fig. 5). As Typhoon Herb traversed northern Taiwan, the airflow over the main body of Taiwan changed from northwesterly to westerly, and then to southwesterly. The moisture-laden typhoon circulation was forced to move over the CMR, and the lifting associated with the upslope motion produced extremely heavy precipitation.

Figure 5 shows the Taiwan topography superimposed on an analysis of the rainfall accumulated during the passage of Herb between 30 July and 2 August. Two major precipitation centers are identified: one at Mountain Nio-Dray in northern Taiwan, with a total precipitation of $1044 \mathrm{~mm}$, and the other at Mount A-Li in central Taiwan, with a record-breaking total accumulation of $1987 \mathrm{~mm}$ over the 2-day period (including a 24-h rainfall of $1094.5 \mathrm{~mm}$ on 31 July, and $892 \mathrm{~mm}$ on 1 August). The heavy rainfall at Mount A-Li is also manifested in the analysis of its hourly rainfall (Fig. 6); over 14 consecutive hours, rainfall in excess of $80 \mathrm{~mm}$ was recorded. In sharp contrast, downslope flow persisted over eastern Taiwan, and very little precipitation was recorded. The downslope flow also produced a topographically induced mesolow over eastern Taiwan near Hualien. According to S.-T. Wang (1997, personal communication), a few hours before landfall,

HERB(1996)

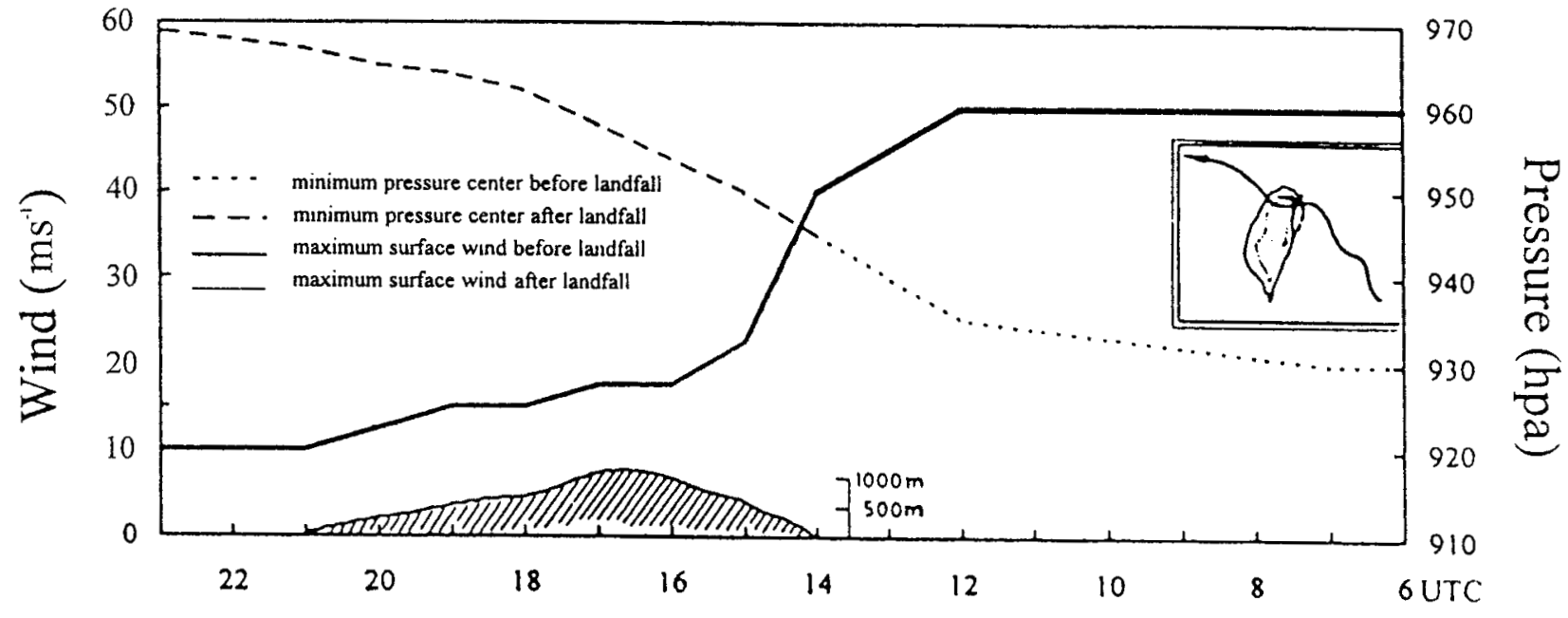

JUL.31

FIG. 4. Measured maximum surface wind and minimum central pressure center as function of time during passage of Typhoon Herb (1996) over Taiwan (after Shieh et al. 1997). 
Typhoon Herb changed its track from a northwestward direction (before 1200 UTC 31 July) to a southwestward direction (after 1200 UTC 31 July). After landfall, the storm changed direction to track northwestward again after 1800 UTC. The Hualien mesolow was generated at around 1800 UTC. This mesolow then moved northward along the east coast of Taiwan.

The heavy rainfall in the central mountain area resulted in numerous devastating landslides and debris flows along the New-Central CrossIsland Highway, leading to heavy loss of both life and property. Several factors that led to such heavy rainfall are summarized as follows.

- Herb was an intense typhoon with a track (making landfall in the northern part of Taiwan) that prevented it from being totally blocked or destroyed by the CMR.

- The circulation of Herb carried an unusually large amount of water vapor that provided the moisture supply for the convective systems embedded within Herb.

- Herb's relatively slow passage $(\sim 7 \mathrm{~h})$ over Taiwan allowed persistent upslope flow on the western slopes of the CMR that resulted in heavy local rainfall over certain geographical areas.

Shieh et al. (1997) indicated that the relation between rainfall and typhoon position (for typhoons with tracks similar to Herb) can be qualitatively described by a schematic diagram with three stages.

- While the typhoon approaches northern Taiwan, the western part of Taiwan experiences an increasing northwesterly flow so that the heavy rainfall occurs in the northern and central areas.

- As the typhoon makes landfall in northern Taiwan, stronger upslope westerly flow in south Taiwan converges with the northwesterly flow in central Taiwan, thus leading to heavy rainfall in the southcentral area of Taiwan. for every $500 \mathrm{~m}$.

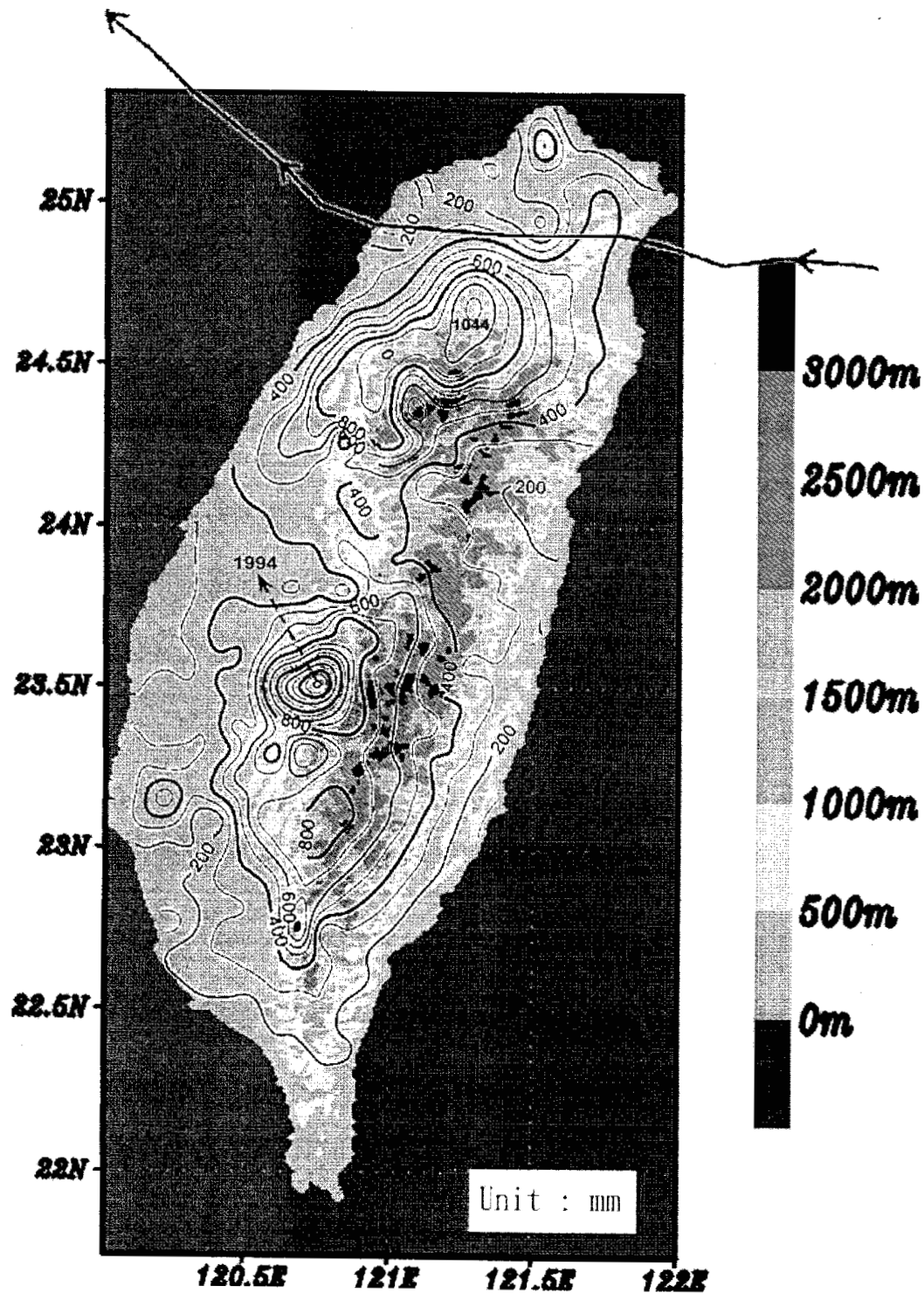

FIG. 5. Accumulated rainfall (mm, with contour interval of $100 \mathrm{~mm}$ ) during passage of Typhoon Herb (30 July-2 August 1996) with track over northeastern Taiwan indicated. Taiwan topography is plotted from 1-km-resolution dataset with shading

- After the typhoon leaves Taiwan, its induced moisture-rich southwesterly flow may bring more rain. Later, as the typhoon center moves farther away from Taiwan, the induced southwesterly flow is nearly parallel to the orientation of the CMR, and precipitation is reduced.

Note that several storms (e.g., Typhoon Gloria of 1963) with a track similar to that of Herb have resulted in similarly heavy rainfall in the central mountain area of Taiwan (Shieh et al. 1995). More studies are needed to improve our understanding of the physical mechanisms that caused the exceptionally heavy rainfall with Herb. 
(a) hourly rainfall (in $\mathrm{mm}$ )

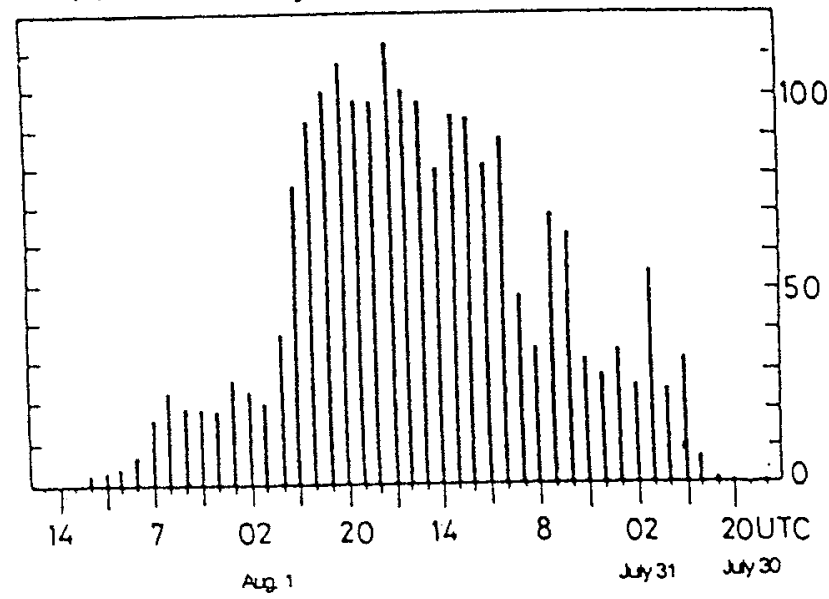

(b)

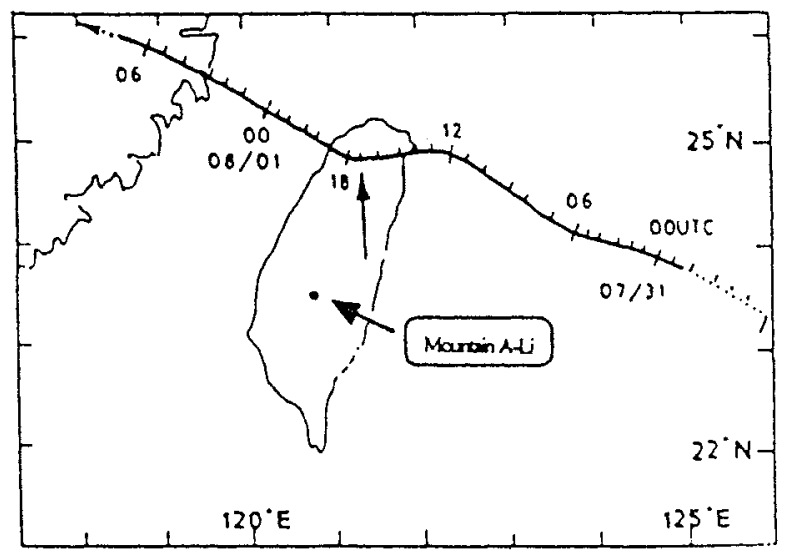

FIG. 6. (a) Hourly rainfall (mm) measured at Mount A-Li station as function of time. Track of Typhoon Herb (1996) (thicker line coresponds to period when hourly rainfall is plotted) and location at Mount A-Li station are indicated in (b) (after Shieh et al. 1997).

\section{c. Model simulation}

The CWB's Typhoon Track Model (45-km resolution) performed well for Typhoon Herb. According to Yeh (1997), the CWB model provided very good track forecasts after 28 July. The average forecast errors were $109 \mathrm{~km}$ for 24 -h and $151 \mathrm{~km}$ for 48 -h forecasts. Yeh (1997) also experimented with a $15-\mathrm{km}$ version of the CWB Typhoon Track Model and produced even better results for track, wind, pressure, and rainfall forecasts.

It is found that, in terms of track prediction, Herb is a relatively easy case for most dynamic and statistical models. For a storm that produced such severe damage in Taiwan, the interest goes beyond an accurate track forecast. For evacuation, flood mitigation, and decisions related to all public activities, accurate forecasts of mesoscale wind and precipitation distribution are needed. The challenging question is, Can a numerical prediction model provide a good rainfall and wind field forecast for a landfalling typhoon of such intensity? Wang $(1980,1989)$ showed that the mesoscale precipitation and wind field distributions during typhoons affecting Taiwan are strongly modulated by the topography of Taiwan. A 45-km grid size is too coarse to resolve the detailed topography of the CMR.

We (also see Kuo and Wang 1997) have experimented with a high-resolution $(6.7 \mathrm{~km})$, nonhydrostatic, mesoscale model (MM5) on the prediction of Typhoon Herb. Our primary interest was to examine the ability of the model to predict the detailed precipitation distribution associated with Typhoon Herb, and to assess the impact of horizontal resolution and detailed topog- raphy on rainfall prediction. Figure 7 shows the daily rainfall prediction and the corresponding model terrain (from 0000 UTC 31 July to 0000 UTC 1 August) from three experiments with resolutions of 6.7, 20, and $60 \mathrm{~km}$, respectively. The highlights of their results are summarized as follows.

1) The 6.7-km MM5 successfully simulated the mesoscale rainfall distribution associated with this extreme rainfall event, although the predicted maximum 24-h rainfall of $818 \mathrm{~mm}$ (Fig. 7a) was about half that observed $(\sim 1700 \mathrm{~mm})$. The MM5 also successfully simulated the subsidence-induced meso low near Hualien.

2) The ability of the model to successfully simulate the observed rainfall declined with the reduction of horizontal grid resolution (as shown by a comparison of Figs. $7 \mathrm{a}-\mathrm{c}$ ). Even with a $20-\mathrm{km}$ grid (Fig. 7b), the model could not reproduce the detailed rainfall distribution, and the model-predicted maximum 24-h rainfall over the CMR and western Taiwan was reduced to $641 \mathrm{~mm}$. The ability of the model to capture the heavy precipitation and its associated mesoscale structure was found to be closely tied to its ability to resolve the detailed topography of the CMR (see Fig. 7d-f).

3) Analysis of the 6.7-km MM5 results showed that the extremely heavy rainfall over the CMR was produced by a steady lifting of a deep layer of moisture-laden atmosphere over the mountains. More than $90 \%$ of the rainfall over the CMR and its slopes was produced by the grid-resolvable scale precipitation physics (figures not shown). 

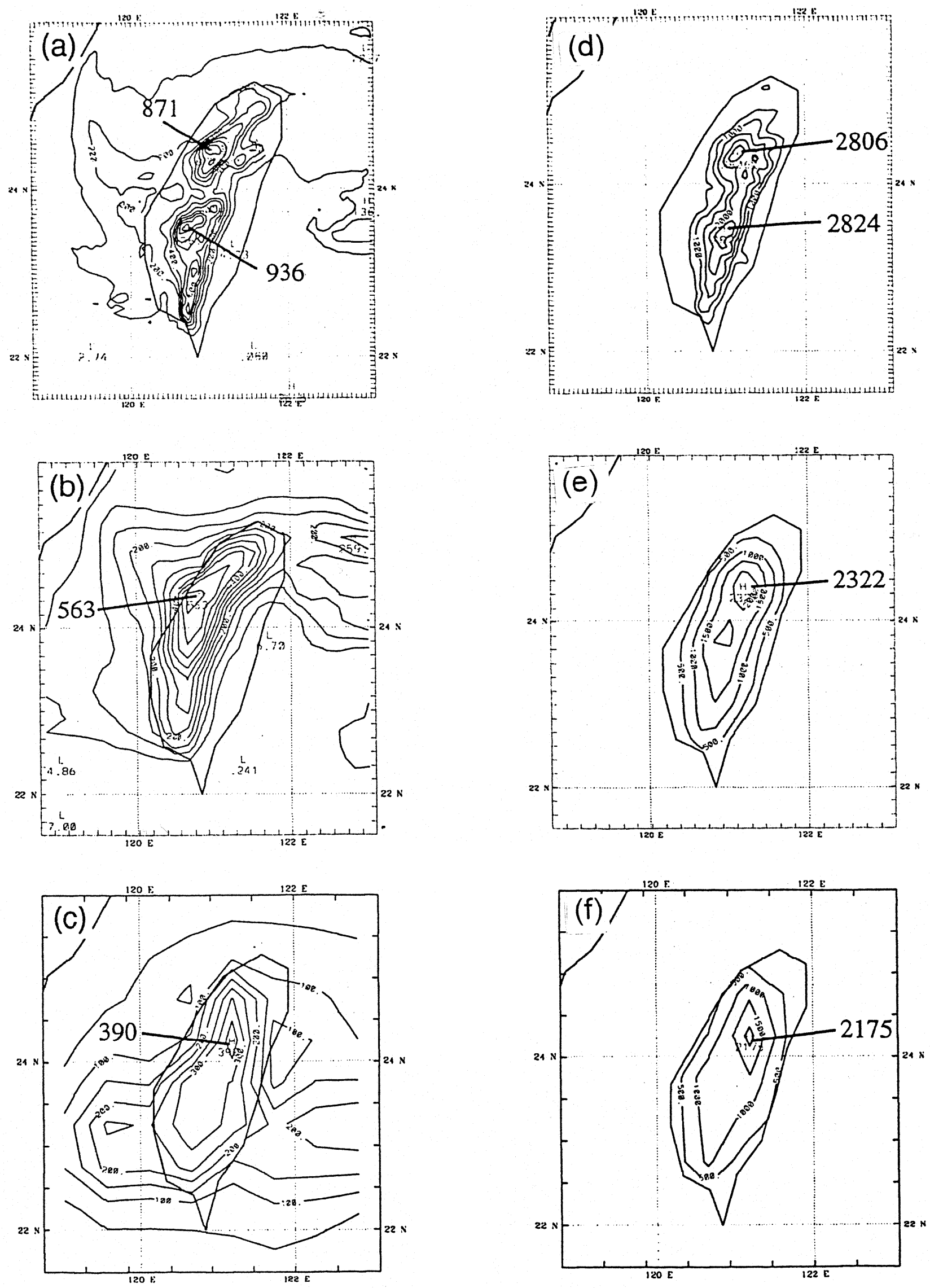
4) The CMR was found to play a key role by both substantially increasing the total rainfall produced by Typhoon Herb (by almost a factor of 3), and by focusing the heavy rainfall over the western slopes of the mountains.

The results presented above show that a highresolution mesoscale model, such as MM5, can be a very useful tool for Taiwan in forecasting the detailed mesoscale precipitation and wind distribution associated with an approaching typhoon. Additional research is required to access the potential of such highresolution mesoscale or typhoon models in order to improve the detailed wind and rainfall forecasts for typhoons near Taiwan.

\section{Workshop on Typhoon Research in the Taiwan Area: 17-18 May 1997}

The workshop was held at the Mesa Laboratory of the National Center for Atmospheric Research, Boulder, Colorado. The aims of this workshop were 1) to review the state of science in typhoon research, with the emphasis on typhoon-topography interaction; 2) to discuss forecasting issues for typhoons approaching Taiwan; and 3) to identify opportunities for collaboration in both basic and forecasting research into typhoons. Participants of the workshop included approximately 25 scientists from Taiwan and 35 scientists invited from the United States, Australia, and China. The workshop was divided into several sessions: Theoretical and Observational Studies of Tropical Cyclones, Modeling Studies of Tropical Cyclones, Forecasting Tropical Cyclones, Aerosonde Experiment 1998, and Planning for Future Research. Excluding the discussions of the problem of hazard mitigation, the major conclusions and recommendations of the workshop can be summarized as follows:

\section{a. Difficulties in the prediction of typhoons near Taiwan \\ 1) Inadequate observations: The current operational observation systems do not provide an accurate de-}

FIG. 7 (preceding page). The 24-h rainfall (mm) ending at 0000 UTC 1 August 1996 from MM5 simulations with highest horizontal resolutions of (a) 6.7, (b) 20, and (c) $60 \mathrm{~km}$. Contour intervals are (a) $100 \mathrm{~mm}$, and $50 \mathrm{~mm}$ for (b) and (c). Corresponding model terrains (m) are shown in (d), (e), and (f), respectively. scription of the structure and intensity of typhoons or the environmental flow associated with them.

2) Insufficient model resolution: Current operational typhoon prediction models have insufficient horizontal resolution to provide detailed descriptions of mesoscale wind and precipitation distribution.

3) Complicated influence of the CMR of Taiwan: The effects of the CMR on the movement and structure of a typhoon are very complicated. Many of these physical processes are not well understood.

\section{b. Bottleneck of typhoon research in Taiwan}

1) Lack of observations: With a lack of mesoscale observations, the three-dimensional dynamic and thermodynamic structures of a typhoon are not properly described before, during, or after it passes over Taiwan. The lack of observations of the fourdimensional evolution of the storm poses a major difficulty in typhoon research.

2) Lack of synergy between observational and modeling work: An interplay between high-resolution numerical simulations and high-resolution mesoscale analysis would offer much insight into the problem of typhoons in Taiwan. Such efforts are rare in the literature.

3) Lack of mesoscale focus: Previous observational and modeling work focused on track prediction, while the main concerns are the mesoscale wind and the precipitation associated with typhoons.

\section{c. Recommended important research topics}

The working group suggested the following three topics as high priority for typhoon research in the Taiwan area.

1) Effects of topography on typhoon motion, mesoscale wind, and precipitation distribution on mesoscale structure of typhoons (in terms of pressure, wind, moisture, temperature, and vorticity), and on the development of secondary vortices.

2) Mesoscale convective systems embedded within typhoons, their interaction with vortex circulation and the CMR, and their impact on wind gusts and rainfall distribution.

3) The priority for typhoon forecasts in Taiwan are listed as 1) track forecast; 2) intensity forecast; 3 ) wind and precipitation forecast. To improve track forecast, the interaction between environmental flow and a typhoon, and the environmental flow's impact on typhoon motion, needs to be better understood. 


\section{d. Other suggestions for applied typhoon research} topics

\section{1) DATA IMPROVEMENT}

As discussed earlier, the improvement in the quantity and quality of data associated with typhoons is highly important to typhoon research and forecasting. Supplementary soundings during the period when a typhoon affects Taiwan, setting up an around-theisland Doppler radar network, an enhanced surface rain gauge network, and an integrated sounding system, as well as the use of aerosondes (Holland et al. 1992) could all bring a lot of useful new data relating to typhoons. Conducting a specific field experiment, with the use of a modern mesoscale observation platform (such as a research aircraft and airborne Doppler radar), to study the four-dimensional evolution of the storm as it interacts with the CMR is of very high priority and should be seriously considered. Meanwhile, the strategy for adaptive observation near the Taiwan area should also be evaluated.

\section{2) STUdy OF THE STATISTICAL AND ANALOG FORECAST METHODS}

The existence of the CMR is a mixed blessing for typhoon forecasting. Because the topography is fixed, if a model can predict the position and intensity of a storm well, the rainfall and wind field distribution can be forecast reasonably well. In other words, as long as the track forecast is correct, the mesoscale precipitation distribution and wind field distribution during typhoons affecting Taiwan are strongly modulated by the topography of Taiwan. Therefore, the statistical and analog method can be very useful for the forecasting of the mesoscale wind and precipitation distribution. One shortcoming of the statistical and analog method is the limited database. One possible approach to increasing the database is to perform high-resolution numerical simulation. By placing typhoons at different locations relative to Taiwan, we can make short-range forecasts of the wind and rainfall distribution. These experiments can be done beforehand and stored for future use. The accuracy and skill of the statistical and analog approaches should be carefully evaluated.

In addition, a high-resolution numerical model could be used to provide "deterministic" forecasting. It is interesting to note that there is a major difference between a 0-24-h forecast and a 24-72-h forecast for typhoons. The focus of a $0-24-h$ forecast is short-range mesoscale prediction of rainfall and wind field distribution, which requires the use of a high-resolution model. For the longer-range forecast, however, the focus is track and intensity prediction. Therefore, a proper vortex specification and an accurate description of the environmental flow are very important, and these, in turn, require the assimilation of all available remote sensing observations (in particular, satellite data) and the use of advanced techniques (such as the four-dimensional variational data assimilation).

\section{3) Model improvement}

Numerical models are the essential tool for typhoon forecasting and research. The workshop participants recommended many areas for research and development in numerical models.

a) Assimilation of satellite data for model initialization. Because of the sparsity of data over the ocean, we should make use of satellite remote-sensing observations. This requires the development of advanced data assimilation systems including 3DVAR (variational data assimilation) and 4DVAR. All available satellite observations including cloud-top winds, water vapor winds, Special Sensor Microwave/Imager rainfall rate, precipitable water observations, as well as scatterometer and Advanced Very High Resolution Radiometer data should be used.

b) Improvement in the methods for vortex implantation (e.g., Kurihara et al. 1995). Vortex specification has a major impact on the performance of a typhoon model. Therefore, improved methods of vortex implantation should be developed and implemented. To improve the implantation procedure, observations from aerosondes may also be used to describe the vortex structure.

c) Model improvement, ensemble forecasting, and high-resolution modeling. Model physics and numerics should be improved. The use of ensemble forecasting techniques and high-resolution mesoscale models should be explored to seek improvement in forecasting skill.

d) Conducting model intercomparison research to assess the advantages and disadvantages of various modeling approaches.

4) INTERACTION BETWEEN SCIENTISTS IN TAIWAN AND OTHER COUNTRIES

Typhoons and hurricanes are essentially the same. Collaboration with the international community can bring new input to the typhoon research program in Taiwan. As an example, scientists in Taiwan should be encouraged to take part in the Hurricane at Landfall (HaL) program. To this end, it should be noted that 
$\mathrm{HaL}$ is one of the three research foci under the U.S. Weather Research Program (see Emanuel et al. 1995; Marks et al. 1998). International scientists should be encouraged to participate in the planning and execution of future typhoon field experiments near Taiwan.

\section{Summary and future directions}

The highly complicated orographic influence on typhoon tracks, circulation, and the associated mesoscale weather systems provides a unique and outstanding research topic in the Taiwan area. The prediction of storm tracks under the influence of the CMR also poses a challenging forecasting problem because of its intimate relationship with the prediction of mesoscale wind and rainfall distribution around the island. This is an interesting and difficult research area worthy of further exploration.

However, as we know, the interaction of a typhoon with the CMR starts when the storm is still several hundred kilometers away from Taiwan. Also, the behavior of a typhoon as a result of terrain interaction is a function of the intensity and size of the typhoon, as well as the environmental flow. Unfortunately, since there are virtually no conventional observations outside the immediate vicinity of Taiwan, our knowledge of the typhoon structure and its environmental flow before the storm comes to Taiwan is extremely limited. This limitation essentially impedes the progress of investigation into the effect of terrain on typhoons.

Due to the limited number of observations, and the very complicated interaction between typhoons and topography, numerical models become a crucial research vehicle to improve our knowledge. However, increased observations are needed for model initialization and verification. In addition, the ability to simulate the detailed typhoon-topography interaction and its associated mesoscale wind and precipitation features by numerical models is still far from perfect, although some progress has been made [e.g., the GFDL hurricane model (Kurihara et al. 1998)].

It is hoped that improved observations from the satellites (e.g., Merrill and Velden 1996), Doppler radars (e.g., Marks et al. 1992), reconnaissance [e.g., the impact of Omega Dropwindsonde (Franklin et al. 1996)], unmanned aircraft (e.g., Holland et al. 1992; Langford and Emanuel 1993), as well as specific field experiments and the GPS/MET system [Global Positioning System/Meteorology (e.g., Ware et al. 1996)], can all be incorporated into high-resolution numeri- cal models through advanced data assimilation techniques. By combining the observational analyses and numerical model experiments, we hope to advance our knowledge of the dynamics of the mountain-typhoon interaction and to use such knowledge to aid typhoon forecasting near Taiwan. It is also hoped that more basic and applied typhoon research can be carried out to improve our understanding of typhoon dynamics, as well as to improve typhoon forecasting in the Taiwan area.

Acknowledgments. The authors would like to thank the National Science Council (NSC) of Taiwan for sponsoring the Workshop on Typhoon Research in the Taiwan Area. Special thanks go to Dr. Ching-Yen Tsay (vice-chairman of NSC) for his support of the workshop, to Mr. Hsinn-Liang Shieh (director general of the Central Weather Bureau of Taiwan), Mr. Shih-Ting Wang, Dr. Wei Wang, and Dr. Wen-Chau Lee for providing data or figures on Typhoon Herb, and to Dr. Cheng-Shang Lee, Dr. HungChi Kuo, and Ms. Ingrid Moore for helping to organize the workshop. We also thank all the participants of the workshop for their contribution.

\section{References}

Batchelor, G. K., 1967: An Introduction to Fluid Dynamics. Cambridge University Press, 615 pp.

Bender, M. A., R. E. Tuleya, and Y. Kurihara, 1985: A numerical study of the effect of a mountain range on a landfalling tropical cyclone. Mon. Wea. Rev., 113, 567-582.

,-- , and -1987 : A numerical study of the effect of island terrain on tropical cyclones. Mon. Wea. Rev., 115, 130-155.

Brand, S., and J. W. Blelloch, 1974: Changes in the characteristics of typhoons crossing the island of Taiwan. Mon. Wea. Rev., 102, 708-713.

Chan, J. C.-L., 1984: An observational study of the physical processes responsible for tropical cyclone motion. J. Atmos Sci., 41, 1036-1048.

Chang, S. W., 1982: The orographic effects induced by an island mountain range on propagating tropical cyclones. Mon. Wea. Rev., 110, 1255-1270.

Chu, K.-K., S.-T. Wang, and H. P. Pao, 1977: Surface wind fields and moving tracks of typhoons when encountering the island of Taiwan. Preprints, 11th Tech. Conf. Hurricane and Tropical Meteorology, Miami Beach, FL, Amer. Meteor. Soc., 84-87.

Clark, T. L., and W. R. Peltier, 1977: On the evolution and stability of finite-amplitude mountain waves. J. Atmos. Sci., 34, 1715-1730.

Emanuel, K. A., and Coauthors, 1995: Report of the first Prospectus Development Team of the U.S. Weather Research Program to NOAA and the NSF. Bull. Amer. Meteor. Soc., 76, 1194 1208.

Franklin, J. L., S. E. Feuer, J. Kaplan, and S. D. Aberson, 1996: Tropical cyclone motion and surrounding flow relations: Searching for beta gyres in Omega Dropwindsonde Datasets. Mon. Wea. Rev., 124, 64-84. 
Holland, G. J., T. McGeer, and H. Youngren, 1992: Autonomous aerosondes for economical atmospheric sounding anywhere on the globe. Bull. Amer. Meteor. Soc., 73, 1987-1998.

Jou, B. J.-D., P.-L. Chang, C.-S. Lee, 1997: Dual Doppler radar analysis of Typhoon Herb (1996). Preprints, Conf. on Weather Analysis and Forecasting, Taipei, Taiwan, Central Weather Bureau, 356-364.

Kuo, Y.-H., and W. Wang, 1997: Rainfall prediction of Typhoon Herb with a mesoscale model. Preprints, Workshop on Typhoon Research in the Taiwan Area. Boulder, CO, National Science Council, 35-45.

Kurihara, Y., M. A. Bender, R. E. Tuleya, and R. J. Ross, 1995: Improvements in the GFDL hurricane prediction system. Mon. Wea. Rev., 123, 2791-2801.

,,--- , and — -1998 : The GFDL hurricane prediction system and its performance in the 1995 hurricane season. Mon. Wea. Rev., 126, 1306-1322.

Langford, J. S., and K. A. Emanuel, 1993: An unmanned aircraft for dropwindsonde development and hurricane reconnaissance. Bull. Amer. Meteor. Soc., 74, 367-375.

Lee, C.-S., S.-T. Wang, S.-L. Shieh, and T.-C. Chen Wang, 1997: Typhoons in the Taiwan area. Preprints, Workshop on Typhoon Research in the Taiwan Area, Boulder, CO, National Science Council, 9-10.

Lin, Y.-L., 1993: Orographic effects on airflow and mesoscale weather systems over Taiwan. Terr., Atmos. Oceanic Sci., 4, 381-420.

Marks, F. D., R. A. Houze, and J. F. Gamache, 1992: Dual-aircraft investigation of the inner core of Hurricane Norbert. Part I: Kinematic structure. J. Atmos. Sci., 49, 919-942.

__ , and Coauthors, 1998: Landfalling tropical cyclones: Forecast problems and associated research opportunities. Bull. Amer. Meteor. Soc., 79, 305-323.

Merrill, R. T., and C. S. Velden, 1996: A three-dimensional analysis of outflow layer of Supertyphoon Flo (1990). Mon. Wea. Rev., 124, 47-63.

Shieh, S.-L., Y.-W. Lin, C.-D. Chen, and C.-Y. Wen, 1995: Study of the characteristics of the precipitation pattern associated with typhoons in the Chia-Nan area (in Chinese). Tech. Rep. NSC 830414-P-052-003-B, National Science Council of Taiwan, 95 pp.

_, S.-T. Wang, M.-D. Cheng, and T.-C. Yeh, 1996: User's guide (1) for typhoon forecasting in the Taiwan area (in Chinese). Res. Rep. CWB84-1M-01, 356 pp.

$-, \ldots, \ldots$, and $—, 1997$ : User's guide (2) for typhoon forecasting in the Taiwan area (in Chinese). Res. Rep. CWB851M-01, $381 \mathrm{pp}$.
Smith, R. B., 1979: The influence of mountains on the atmosphere. Advances in Geophysics, Vol. 21, Academic Press, 87-230.

_ 1989a: Mountain-induced stagnation points in hydrostatic flow. Tellus, 41A, 270-274.

_ 1989b: Comment on "Low-Froude-number flow past threedimensional obstacles. Part I: Baroclinically generated lee vortices." J. Atmos. Sci., 46, 3611-3613.

— mountains in shallow-water flow with a drifting vortex. $J$. Atmos. Sci., 52, 436-454.

Smolarkiewicz, P., and R. Rotunno, 1989: Low-Froude number flow past three-dimensional obstacles. Part I: Baroclinically generated lee vortices. J. Atmos. Sci., 46, 1154-1164.

Wang, S.-T., 1980: Prediction of the behavior and strength of typhoons in Taiwan and its vicinity. Res. Rep. 108, National Science Council, Taipei, Taiwan, 100 pp.

_ 1989: Observational analysis of the orographically induced disturbances during TAMEX. Workshop on TAMEX Preliminary Scientific Results, Taipei, Taiwan, National Science Council, 279-286.

Wang, T.-A., C.-C. Wu, and Y.-L. Lin, 1997: An idealized numerical study of the effects of Taiwan topography on typhoons. Preprints, Conf. on Weather Analysis and Forecasting, Taipei, Taiwan, Central Weather Bureau, 190-198.

Ware, R., and Coauthors, 1996: GPS sounding of the atmosphere from low Earth orbit: Preliminary results. Bull. Amer. Meteor. Soc., 77, 19-40.

Wu, C.-C., and Y. Kurihara, 1996: A numerical study of the feedback mechanisms of hurricane-environment interaction on hurricane movement from the potential vorticity perspective. J. Atmos. Sci., 53, 1398-1416.

- , and Y.-T. Cho, 1997: A numerical study of the effect of Taiwan terrain on Typhoon Gladys (1994). Preprints, 22d Conf. on Hurricanes and Tropical Meteorology, Fort Collins, CO, Amer. Meteor. Soc., 240-241.

Yeh, T.-C., 1997: A review of the typhoon forecast in CWB and the performance of CWB Typhoon Track Model. Preprints, Workshop on Typhoon Research in the Taiwan Area, Boulder, CO, National Science Council, 46-49.

— Taiwan orography. Part I: Upstream track deflections. Mon. Wea. Rev., 121, 3193-3212.

$\longrightarrow$, and $-1993 \mathrm{~b}$ : Interaction of typhoons with the Taiwan orography. Part II: Continuous and discontinuous tracks across the island. Mon. Wea. Rev., 121, 3213-3233.

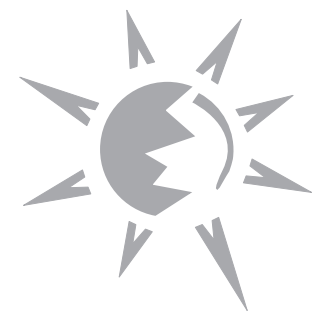

